\title{
Ten-Year Outcomes Of Intensity-Modulated Radiotherapy (IMRT) Combine With Chemotherapy Versus IMRT Alone For Stage II Nasopharyngeal Carcinoma In The Real-World Study (RWD)
}

This article was published in the following Dove Press journal:

Cancer Management and Research

\author{
Xing-Chen Ding ${ }^{1,2, *}$ \\ Ping-Ping Fan $^{3, *}$ \\ Peng Xie (iD ${ }^{2}$ \\ Bing-Jie Fan ${ }^{2}$ \\ Jia Yang ${ }^{2}$ \\ Li-Yang Jiang ${ }^{2}$ \\ Xin-Bin Bai ${ }^{1,2}$ \\ Jin-Ming $\mathrm{Yu}^{2}$ \\ Man $\mathrm{Hu}^{2}$
}

'Department of Oncology, School of Medicine and Life Sciences, University of Jinan-Shandong Academy of Medical Sciences, Jinan, People's Republic of China; ${ }^{2}$ Department of Radiation Oncology, Shandong Cancer Hospital and Institute, Shandong First Medical University and Shandong Academy of Medical Sciences, Jinan, Shandong, People's Republic of China; ${ }^{3}$ Department of Radiology, The Affiliated Hospital of Qingdao University, Qingdao, People's Republic of China

*These authors contributed equally to this work

Correspondence: Man Hu Department of Radiation Oncology, Shandong Cancer Hospital and Institute, Shandong First Medical University and Shandong Academy of Medical Sciences, Jinan 250I I7, People's Republic of China

Tel +86-53I-67626I52

Fax +86-53I-67626I53

Email human5770@I63.com
Objectives: The aim was to define the role of chemotherapy in stage II nasopharyngeal carcinoma (NPC) and to identify the toxicity of chemotherapy for these patients in the era of intensity-modulated radiotherapy (IMRT).

Methods: Between January 2002 and December 2013, 169 patients with stage II NPC were analyzed. Of these patients, 149 patients treated with chemotherapy were divided into three groups as follows: neoadjuvant chemotherapy followed by IMRT (NCT) group, concurrent chemotherapy with IMRT (CCRT) group, and neoadjuvant chemotherapy followed by CCRT $(\mathrm{NC}+\mathrm{CCRT})$ group. In addition, 20 patients received IMRT alone. We retrospectively assessed the 10-year survival and acute adverse effects in the patients using SPSS software. Results: The median follow-up time was 93 months (2-160 months). The 10-year OS of the NCT, CCRT, NC+CCRT groups vs the IMRT alone group was $69.8 \%, 63.4 \%, 69.7 \%$ vs $72.4 \%$, respectively ( $P=0.664,0.940$, and 0.998 , respectively). Both univariable and multivariable analyses showed that the addition of chemotherapy to IMRT did not significantly improve the 10-year survival outcomes. The hematotoxicity and mucous reaction of patients with chemotherapy were more serious than those with IMRT alone $(P=0.007$ and 0.049$)$. Distant metastasis for stage II NPC patients mostly occurred within 3 years, which is very different from patients with advanced NPC.

Conclusion: Patients with stage II NPC who are treated with IMRT may obtain satisfactory long-term survival outcomes. The additional chemotherapy cannot significantly increase survival; however, it may remarkably increase treatment-associated acute toxic reactions.

Keywords: stage II nasopharyngeal carcinoma, NPC, intensity-modulated radiotherapy, IMRT, chemotherapy

\section{Introduction}

Nasopharyngeal carcinoma (NPC), characterized by its unique geographic distribution, is endemic in the eastern and southeastern regions of Asia. Approximately $80 \%$ of NPC cases occur in People's Republic of China. ${ }^{1}$ NPC is more sensitive to radiotherapy (RT) than other head and neck carcinomas due to its specific biological characteristics. As a consequence, RT has been the basic curative treatment of NPC. Although NPC is also highly chemosensitive, the benefit of RT varies in different stages. For stage I NPC, it has been reported that RT as a single modality achieves an excellent outcome with a $>95 \%$ overall survival (OS) at 10 years. $^{2}$ For 
patients with stage III-IVB, combined chemotherapy and RT has become a standard treatment due to sufficient evidence showing improved OS from $50 \%$ to $70 \%$ compared to RT alone. ${ }^{3-6}$ To date, it is still controversial if chemotherapy is beneficial for patients with stage II NPC.

In the traditional RT era, several studies demonstrated that the addition of chemotherapy to RT improves the clinical outcomes for stage II NPC. ${ }^{2,7-9}$ As the RT technique advances rapidly, intensity-modulated radiotherapy (IMRT) has replaced traditional RT to become the standard treatment technique for NPC. Previous studies have reported that IMRT alone has acquired superb outcomes for stage II NPC. ${ }^{10-16}$ Currently, the necessity of IMRT combined with chemotherapy for stage II NPC is questioned. The National Comprehensive Cancer Network and European Society for Medical Oncology recommend concurrent chemoradiotherapy with or without adjuvant chemotherapyas the standard basic treatment for stage II-IVB patients. However, AC is mainly used in patients with residual disease or those with advanced disease due to the lower compliance rate, especially after concurrent chemoradiotherapy. ${ }^{17,18}$ Evidence from a Phase III trial supports that concurrent chemoradiotherapy is recommended for some early stages of NPC, ${ }^{19}$ which comprises the level $2 \mathrm{~B}$ evidence in the latest version of guidelines. ${ }^{20}$ Interestingly, neoadjuvant chemotherapy plus concurrent chemoradiotherapy has been commonly used in stage IIIVB NPC patients in many hospitals in People's Republic of China because the considerable response rate is superb. Nevertheless, this combined treatment was recommended as level 3 evidence in the latest version of guidelines. ${ }^{20} \mathrm{~A}$ pooled data analysis of two Phase III studies has shown that the addition of neoadjuvant chemotherapy to RT significantly improves the disease-specific survival of stage II-IVB NPC patients but that no improvement in OS is observed. $^{21}$ The efficacy of neoadjuvant chemotherapy remains unclear, and data for stage II NPC patients are limited in the IMRT era. Until recently, almost all previous studies have demonstrated that IMRT combined with chemotherapy (IMRT $+\mathrm{CT})$ does not improve survival for stage II NPC patients but instead increases the acute toxicity reaction. ${ }^{22-25}$ In these studies, however, the median reported follow-up was $2-5$ years, which is relatively short for early-stage patients with NPC.

In our practice, the majority of patients with NPC received IMRT after 2002. Therefore, we retrospectively reviewed the long survival outcome of patients with stage II NPC who were treated with IMRT with or without chemotherapy. The aim of the study was to investigate the roles of chemotherapy for stage II NPC patients and to further evaluate the efficacy of different chemotherapy patterns in treatments during the IMRT era.

\section{Patients And Methods Patients And Workup}

This study was approved by the Institutional Review Board of Shandong Cancer Hospital and Institute, Shandong First Medical University and Shandong Academy of Medical Sciences (no SDTHEC201806036). All procedures were in accordance with the principles of the Declaration of Helsinki and its later amendments or comparable ethical standards. Due to the retrospective nature of the study, informed consent was waived by the Ethics Committee. Between January 2002 and December 2013, 2647 patients were histologically diagnosed with NPC at the Shandong Cancer Hospital. Pretreatment evaluation included complete blood chemistry, fiberoptic endoscopic examination of the nasopharynx, contrast-enhanced computed tomography and/or magnetic resonance imaging (MRI) of the head-and-neck region to evaluate the primary tumor and regional lymph nodes. Chest computed tomography, bone scintigraphy, ultrasonography of the abdominal region and/or fluorine-18 fluorodeoxyglucose positron emission tomography-computed tomography (18-FDG PET/CT) were used to diagnosis distant metastases. All patients were reviewed and restaged according to the 8th edition AJCC classification system. Two hundred forty-four patients were classified as having stage II NPC. Of the stage II patients, 75 were excluded due to the following reasons: inadequate dose of RT ( $<66 \mathrm{~Gy}$ ); age $<18$ or $>80$ years old; second primary tumor; previous chemotherapy history and RT of neck region history; or traditional RT. Finally, the remaining 169 patients were analyzed in the study. All patients were divided into the $\mathrm{T}_{2} \mathrm{~N}_{0} \mathrm{M}_{0}$ and $\mathrm{T}_{1-2} \mathrm{~N}_{1} \mathrm{M}_{0}$ subgroups.

\section{Radiotherapy}

All patients received computed tomography simulation with intravenous injection contrast to delineate the target volume, and the head, neck, and shoulder of all patients were immobilized by a thermoplastic mask. Target volumes include gross tumor volume (GTV), clinical target volume (CTV), and planning target volume (PTV). $\mathrm{GTV}_{66-72}$ (the subscript 66-72 denotes the radiation dose delivered) includes primary tumor and metastatic lymph nodes. Different regions need accurate selection and 
delineation of the CTV. The high-risk region $\left(\mathrm{CTV}_{54-60}\right)$ was defined as the entire nasopharynx, ensuring inferior coverage of soft palate, clivus, skull base, pterygoid fossae, parapharyngeal space, sphenoid sinus, posterior $1 / 3$ of the maxillary sinuses, posterior $1 / 3$ of the nasal cavity, posterior ethmoid sinuses, and retropharyngeal nodal regions in levels II-V. When the lymph nodes in level II were larger than $3 \mathrm{~cm}$ or had extranodal extension, level IB nodal region was included. The low-risk region $\left(\mathrm{CTV}_{44-50}\right)$ was the cervical lymph node prevention area. PTV was delineated by adding $3 \mathrm{~mm}$ margins around CTVs to setup the variability and internal motion. If CTVs were adjacent to critical organs, such as brain stem and spinal cord, the margin was reduced to $1 \mathrm{~mm}$. The organs at risk, including the brainstem, spinal cord, optic nerves, optic chiasm, pituitary gland, lens, temporal lobes, parotid glands, temporomandibular joints, and mandible, were also delineated. All patients were administered 1.8-2 Gy/d for 5 days a week.

\section{Chemotherapy}

Chemotherapy was used in neoadjuvant and concurrent settings. Accordingly, there were four treatment groups as follows: IMRT alone in 20 patients; neoadjuvant chemotherapy followed by IMRT (NCT) in 25 patients; concurrent chemotherapy with IMRT (CCRT) in 55 patients; and neoadjuvant chemotherapy followed by CCRT $(\mathrm{NC}+\mathrm{CCRT})$ in 69 patients. Among the patients treated with chemotherapy, 96 patients used the PF regimen as follows: cisplatin (75-100 $\mathrm{mg} / \mathrm{m}^{2}$ intravenously in three daily doses) plus 5-fluorouracil (500-1000 mg/m $\mathrm{m}^{2}$ intravenously in five daily doses). In addition, 27 patients received the TP regimen as follows: cisplatin (75-100 mg/m² intravenously in three daily doses) plus paclitaxed $\left(135 \mathrm{mg} / \mathrm{m}^{2}\right.$ intravenously on day 1$)$. Of patients who underwent concurrent chemotherapy, 34 received cisplatin only $\left(75-100 \mathrm{mg} / \mathrm{m}^{2}\right.$ intravenously in three daily doses), 16 received the TP regimen, and 40 received the $\mathrm{PF}$ regimen. The reason that patients received chemotherapy mainly for patients with advanced $\mathrm{N}$, larger tumor volume, or at the physician's discretion and so on (the record was incomplete due to retrospective nature).

\section{Management Of Acute Toxicity Reactions}

Treatment-related toxicity was scored according to the Radiation Therapy Oncology Group radiation morbidity scoring criteria. The acute toxicity mainly included skin reaction, mucous reaction, and hematotoxicity. Hematotoxicity mainly included leucopenia, neutropenia, thrombopenia, and oligocythemia. Routine blood work was performed at least once a week, and biochemical analysis was performed at least once every 2 weeks during the course of treatment. The acute RT reactions were evaluated once a week from the start of RT to 3 months after the end of RT.

\section{Follow-Up}

After completing treatment, patients were subsequently followed up every 3 months during the first 2 years and every 6 months during the second year to the fifth year, and patients were then followed up every year until death. Basic blood chemistry, computed tomography and/or MRI (including base of skull, nasopharynx, and neck to clavicles) as well as chest radiography, computed tomography and abdomen ultrasonography were performed. For patients with evidence of local-regional recurrence or distant metastasis, biopsy, pathological diagnosis, and/or FDG PET-CT were required to confirm disease progression.

The OS was defined as the period from the start of treatment to the date of death from any cause or the censoring of patients at the last follow-up. Progressionfree survival (PFS) was defined as the period from the start of treatment to the first progression or final follow-up. Local-regional relapse-free survival (LRFS) was defined as the period from the start of treatment to first localregional recurrence or final follow-up. Distant metastasisfree survival (DMFS) was defined as the interval from the start of treatment to first distant metastasis or final follow-up.

\section{Statistical Analysis}

SPSS for Windows (version 20.0; IBM Corporation, NY, USA) was used to perform all statistical analyses. Chisquare test or Fisher exact test was performed to compare the difference of basic characteristics (age, sex, $\mathrm{N}$ stage, $\mathrm{T}$ stage, pathology (WHO), and clinical stage) among patients treated by NCT, CCRT, NC+CCRT, and IMRT alone. Nonparametric tests were used to compare acute toxicity reactions among treatment arms. Survival curves were calculated using the Kaplan-Meier method and were analyzed by the log-rank test. The Cox proportional hazard model using backward stepwise elimination procedure to remove variables with a p-value of $\geq 0.10$ was used in multivariate analysis to determine the prognostic significance of variables. Considering the imbalance date between different groups, a bootstrap validator was used during the analyses to revise the statistical bias. ${ }^{26}$ All 
statistical tests were two-sided, and $P<0.05$ was considered statistically significant.

\section{Results}

\section{Patient And Tumor Characteristics}

The study population consisted of 126 males and 43 females, and the median age was 46 years old (range from 18 to 78 years). The patients received the following treatments: 149 patients received IMRT $+\mathrm{CT} ; 63.1 \%$ of patients (94/149) received neoadjuvant chemotherapy; and $88.6 \%$ of patients $(124 / 140)$ received concurrent chemotherapy. In a subgroup, there were 47 patients staged in $\mathrm{T}_{2} \mathrm{~N}_{0} \mathrm{M}_{0}$, and the other 122 were staged in $\mathrm{T}_{1-2} \mathrm{~N}_{1} \mathrm{M}_{0}$. The clinical characteristics of patients were compared according to IMRT with or without chemotherapy (Table 1). The characteristics of patients in the different groups were in good balance, except for $\mathrm{N}$ stage and clinical stage. There were significantly more patients with $\mathrm{N} 1$ stage and $\mathrm{T}_{2} \mathrm{~N}_{1} \mathrm{M}_{0}$ stage in the IMRT $+\mathrm{CT}$ groups compared to the IMRT alone group (NCT, CCRT, and NC+CCRT vs IMRT alone; $\mathrm{N}$ stage: $12.4 \%, 20.1 \%$, and $34.4 \%$ vs $5.3 \%$, respectively, $\mathrm{P}=0.001$; and $\mathrm{T}_{2} \mathrm{~N}_{1} \mathrm{M}_{0}$ stage: $11.8 \%, 18.3 \%$, and $33.8 \%$ vs $5.3 \%$, respectively, $\mathrm{P}=0.001$ ).

\section{Survival}

By the end of December 2017, the median follow-up was 93 months (2-160 months) with the following stats: 43 patients died, 7 patients experienced loco-regional recurrence, and 5 patients developed distant metastases. The total 10-year OS, PFS, LRFS, and DMFS were $70.8 \%$, $87.4 \%, 87.4 \%$, and $87.4 \%$, respectively. The survival curves are shown in Figure 1. No statistically significant differences in the 10-year OS, PFS, and LRFFS were found in the NCT, CCRT, and NC+CCRT groups compared to the IMRT alone group (OS: 69.8\%, 63.4\%, and $69.7 \%$ vs $72.4 \%, \mathrm{P}=0.664,0.940$, and 0.998 , respectively; PFS: $74.1 \%, 93.8 \%$, and $89.3 \%$ vs $75.5 \%, \mathrm{P}=0.620,0.169$, and 0.156 , respectively; LRFS: $74.1 \%, 93.8 \%$, and $89.3 \%$ vs $75.5 \%, \mathrm{P}=0.620,0.169$, and 0.156 , respectively; DMFS: $74.1 \%, 98 \%$, and $96.5 \%$ vs $100 \%, \mathrm{P}=0.170,0.552$, and 0.452 , respectively) as shown in Table 2.

Table 3 shows the results of univariate and multivariate analyses of prognostic factors. In the univariate analyses,

Table I Baseline Characteristics Of The 169 Patients With Stage II NPC

\begin{tabular}{|c|c|c|c|c|c|c|}
\hline Characteristics & $\begin{array}{l}\text { Total } \\
(n=169,100 \%)\end{array}$ & $\begin{array}{l}\text { IMRT Alone } \\
(n=20,11.8 \%)\end{array}$ & $\begin{array}{l}\text { NCT } \\
(n=25,14.8 \%)\end{array}$ & $\begin{array}{l}\text { CCRT } \\
(n=55,32.5 \%)\end{array}$ & $\begin{array}{l}\text { NC+CCRT } \\
(n=69,40.9 \%)\end{array}$ & $P$ \\
\hline $\begin{array}{l}\text { Age (median age 46-year) } \\
\quad \leq 46 \\
\quad>46\end{array}$ & $\begin{array}{l}80(47.3 \%) \\
89(52.7 \%)\end{array}$ & $\begin{array}{l}7(4.1 \%) \\
13(7.7 \%)\end{array}$ & $\begin{array}{l}13(7.7 \%) \\
12(7.1 \%)\end{array}$ & $\begin{array}{l}21(12.4 \%) \\
34(20.1 \%)\end{array}$ & $\begin{array}{l}39(23.1 \%) \\
30(17.8 \%)\end{array}$ & 0.131 \\
\hline $\begin{array}{l}\text { Sex } \\
\qquad \text { Male } \\
\text { Female }\end{array}$ & $\begin{array}{l}126(74.6 \%) \\
43(25.5 \%)\end{array}$ & $\begin{array}{l}17(10.1 \%) \\
3(1.7 \%)\end{array}$ & $\begin{array}{l}15(8.9 \%) \\
10(5.9 \%)\end{array}$ & $\begin{array}{l}42(24.9 \%) \\
13(7.6 \%)\end{array}$ & $\begin{array}{l}52(30.8 \%) \\
17(10.1 \%)\end{array}$ & 0.279 \\
\hline $\begin{array}{l}\text { Pathology (WHO) } \\
\text { II type } \\
\text { III type }\end{array}$ & $\begin{array}{l}81(47.9 \%) \\
88(52.1 \%)\end{array}$ & $\begin{array}{l}7(4.1 \%) \\
13(7.7 \%)\end{array}$ & $\begin{array}{l}\text { I4 (8.3\%) } \\
\text { II (6.5\%) }\end{array}$ & $\begin{array}{l}33(19.5 \%) \\
22(13.0 \%)\end{array}$ & $\begin{array}{l}27(16.0 \%) \\
42(24.9 \%)\end{array}$ & 0.062 \\
\hline $\begin{array}{l}\text { T stage } \\
\text { TI } \\
\text { T2 }\end{array}$ & $\begin{array}{l}5(3 \%) \\
164(97 \%)\end{array}$ & $\begin{array}{l}0(0.0 \%) \\
20(11.8 \%)\end{array}$ & $\begin{array}{l}\text { I (0.6\%) } \\
24(14.2 \%)\end{array}$ & $\begin{array}{l}3(1.8 \%) \\
52(30.8 \%)\end{array}$ & $\begin{array}{l}\text { I (0.6\%) } \\
68(40.3 \%)\end{array}$ & 0.485 \\
\hline $\begin{array}{c}\text { N } \text { stage }^{a} \\
\text { No } \\
\text { NI }\end{array}$ & $\begin{array}{l}47(27.8 \%) \\
122(72.2 \%)\end{array}$ & $\begin{array}{l}\text { II (6.5\%) } \\
9(5.3 \%)\end{array}$ & $\begin{array}{l}4(2.4 \%) \\
21(12.4 \%)\end{array}$ & $\begin{array}{l}21 \text { (12.4\%) } \\
34(20.1 \%)\end{array}$ & $\begin{array}{l}\text { II (6.5\%) } \\
58(34.4 \%)\end{array}$ & $0.001 *$ \\
\hline $\begin{array}{c}\text { Clinical stage }^{\mathrm{a}} \\
\text { TINIM0 } \\
\text { T2NOM0 } \\
\text { T2NIM0 }\end{array}$ & $\begin{array}{l}5(3 \%) \\
47(27.8 \%) \\
117(69.2 \%)\end{array}$ & $\begin{array}{l}0(0.0 \%) \\
\text { II (6.5\%) } \\
9(5.3 \%)\end{array}$ & $\begin{array}{l}\mathrm{I}(0.6 \%) \\
4(2.4 \%) \\
20(11.8 \%)\end{array}$ & $\begin{array}{l}3(1.8 \%) \\
2 I(12.4 \%) \\
31(18.3 \%)\end{array}$ & $\begin{array}{l}\text { I }(0.6 \%) \\
\text { II }(6.5 \%) \\
57(33.8 \%)\end{array}$ & $0.00 I^{*}$ \\
\hline
\end{tabular}

Notes: according to the eighth edition AJCC/UICC staging system. *Means $P \leq 0.05$.

Abbreviation: IMRT, intensity-modulated radiotherapy; NCT, neoadjuvant chemotherapy followed by RT alone; CCRT, concurrent chemoradiotherapy; NC+CCRT, neoadjuvant chemotherapy followed by CCRT; T, primary tumor; $\mathrm{N}$, lymph node; n, number. 


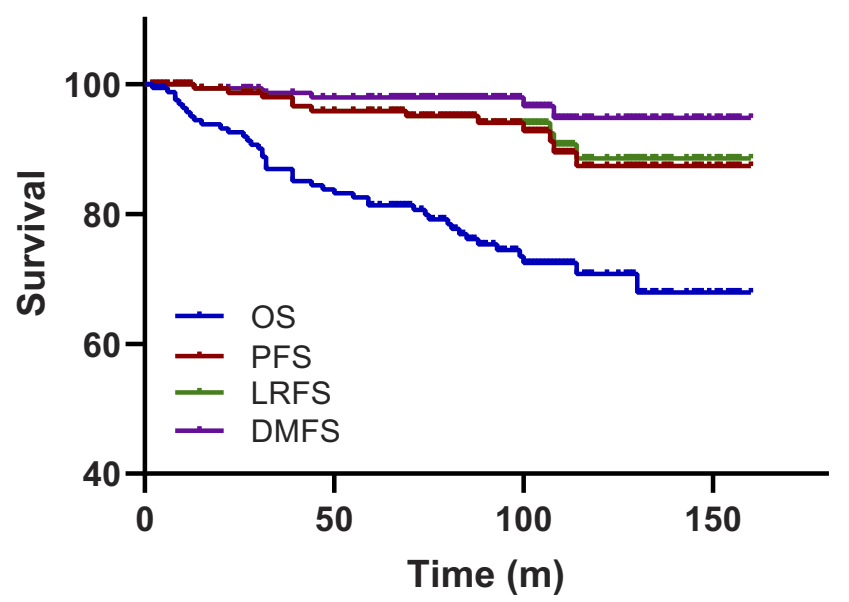

Figure I Overall survival (OS), progression-free survival (PFS), local recurrence-free survival (LRFS), and distant metastasis-free survival (DMFS) rates for all patients.

age and $\mathrm{N}$ stage were significant prognostic factors for OS of stage II patients ( $P=0.007$ and $P=0.035$, respectively). No significant prognostic factor was identified for PSF, LRFS, or DMFS. Moreover, the results of the multivariable analyses were in line with the outcome of the univariable analyses. The OS tended to decrease with $\mathrm{N} 1$ stage (Figure 2A) and older age (Figure 2B) (age: HR 2.686, 95\% CI 1.347-5.353, $P=0.005$; and N stage: HR $3.065,95 \%$ CI 1.238-7.588, $P=0.015$ ).

Upon subgroup analysis, we further analyzed whether adding chemotherapy to IMRT can improve survival benefits in the $\mathrm{T}_{1-2} \mathrm{~N}_{1} \mathrm{M}_{0}$ group. In the $\mathrm{T}_{1-2} \mathrm{~N}_{1} \mathrm{M}_{0}$ group, neoadjuvant chemotherapy with concurrent chemoradiotherapy significantly improved PFS (86.4\% vs 60\%, $P=0.037$ ) (Figure $3 \mathrm{~A}$ and Table 2). Adding neoadjuvant chemotherapy, concurrent chemoradiotherapy or both to IMRT significantly improved the LRFS (NCT, CCRT, and $\mathrm{NC}+\mathrm{CCRT}$ vs IMRT alone: 100\%, 92.6\%, and $90.3 \%$ vs $60 \%, \mathrm{P}=0.02,0.034$, and 0.007 , respectively) (Figure 3B and Table 2). However, no statistically significant difference in OS and DMFS was found between the IMRT $+\mathrm{CT}$ groups and the IMRT group. The results of the multivariate analyses for the $\mathrm{T}_{1-2} \mathrm{~N}_{1} \mathrm{M}_{0}$ subgroup are shown in Table 3 . The results of the multivariate analyses for the $\mathrm{T}_{1-2} \mathrm{~N}_{1} \mathrm{M}_{0}$ subgroup are shown in Table 3. Older age was associated with poor OS (HR 2.589, 95\% CI 1.243-5.392, $P=0.011)$. Neoadjuvant chemotherapy and concurrent chemotherapy were dependent prognostic factors for PFS (NCT: HR 0.320, 95\% CI 0.102-1.002, $\mathrm{P}=0.05$; CCRT: HR 0.207 , 95\% CI $0.055-0.775$, $\mathrm{P}=0.019$ ) and LRFS (NCT: HR 0.121, 95\% CI 0.0230.628, $\mathrm{P}=0.012$; CCRT: HR 0.093, 95\% CI 0.011-0.780,
$\mathrm{P}=0.029$ ). None of the tested factors was found to be prognostic for DMFS.

\section{Toxicity Reaction}

No fatal toxicity reaction occurred in the patients. Fifty-nine patients suffered $\geq$ grade 3 hematotoxicity with $98.3 \%(58 / 59)$ occurring in the IMRT $+\mathrm{CT}$ groups. Seventeen patients suffered $\geq$ grade 3 skin reaction with $94.1 \%$ (16/17) occurring in the IMRT+CT groups. Sixty patients suffered $\geq$ grade 3 mucous reaction with $91.7 \%(55 / 60)$ occurring in the IMRT + CT groups (Table 4). The hematotoxicity and mucous reaction of patients in the IMRT $+\mathrm{CT}$ groups were much more serious than those patients treated with IMRT alone $(P=0.007$ and 0.049 , respectively) (Table 4 ). Table 4 shows the higher incidence of skin reaction in the IMRT $+\mathrm{CT}$ groups than the IMRT alone group, but there were no statistical differences between the groups $(P=0.173)$. In the $\mathrm{T}_{1-2} \mathrm{~N}_{1} \mathrm{M}_{0}$ subgroup, the same results were obtained. The incidence of $\geq$ grade 3 hematotoxicity and mucositis was significantly higher in patients treated with IMRT $+\mathrm{CT}$ than in those treated with IMRT alone ( $P=0.033$ and 0.022 , respectively), but there was no difference in skin reaction.

\section{Discussion}

The present study showed that combining neoadjuvant chemotherapy, concurrent chemotherapy or both with IMRT did not improve survival outcomes in stage II NPC patients, but the combination treatments increased the incidences of acute treatment-associated toxicities compared to treatment with IMRT alone. Furthermore, subgroup analysis showed that IMRT combined with chemotherapy had more effective loco-regional control than IMRT alone in patients in the $\mathrm{T}_{1-2} \mathrm{~N}_{1} \mathrm{M}_{0}$ subgroup.

Although many studies have documented the survival outcomes in early-stage NPC, there have been few studies reporting the outcomes with 10-year follow-up, especially for NPC patients with stage II. To the best of our knowledge, the present study was the first to report the longest follow-up time in stage II NPC patients treated by IMRT. With a median follow-up of 93 months, 169 patients with stage II had excellent survival outcome. Using traditional RT in a retrospective study with long-term follow-up, the 5year and 10-year disease-specific survival rates were 77\% and $60 \%$, the LRFS values were $83 \%$ and $78 \%$, and the DMFS values were $72 \%$ and $64 \%$, respectively, ${ }^{27}$ which were lower than in patients treated with IMRT. The worse OS and loco-regional control of traditional RT may be due to the underdosing of tumor with the goal of normal tissue 


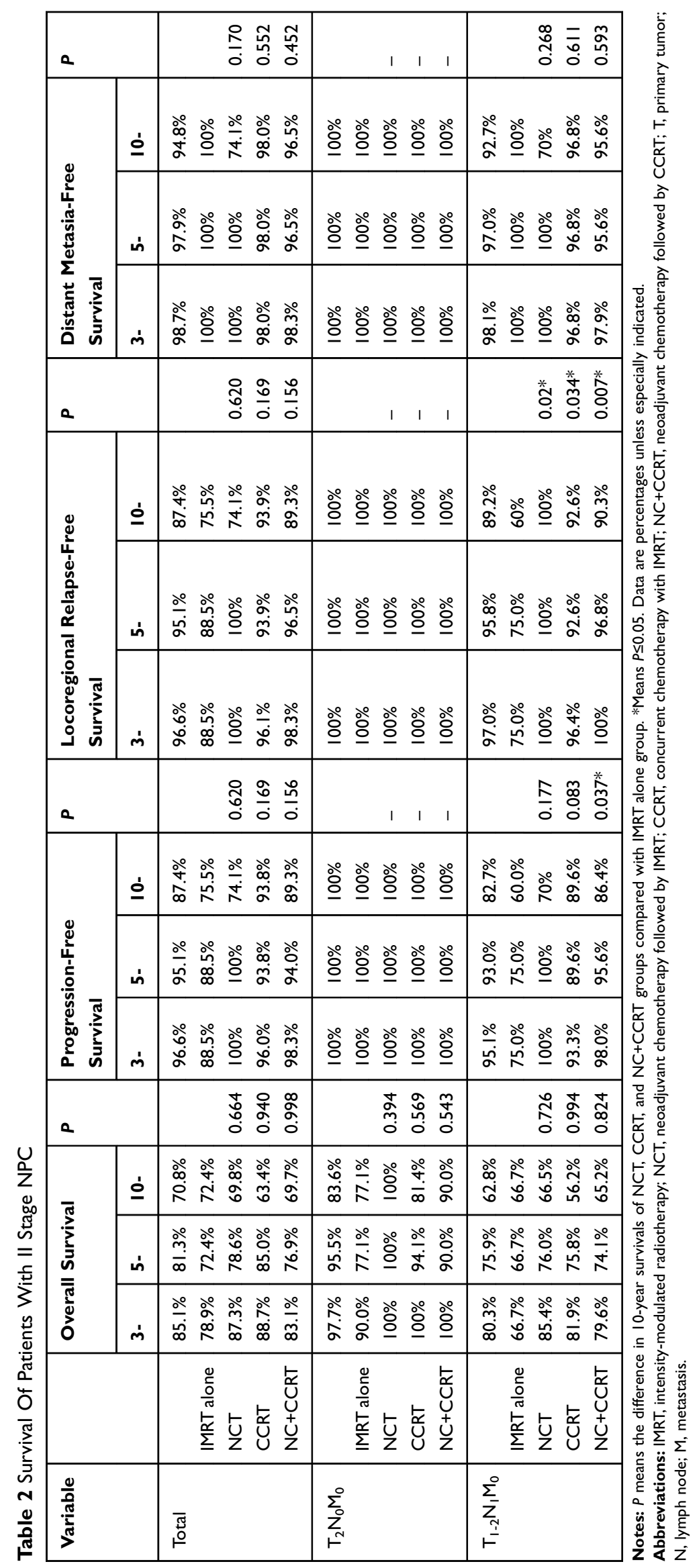




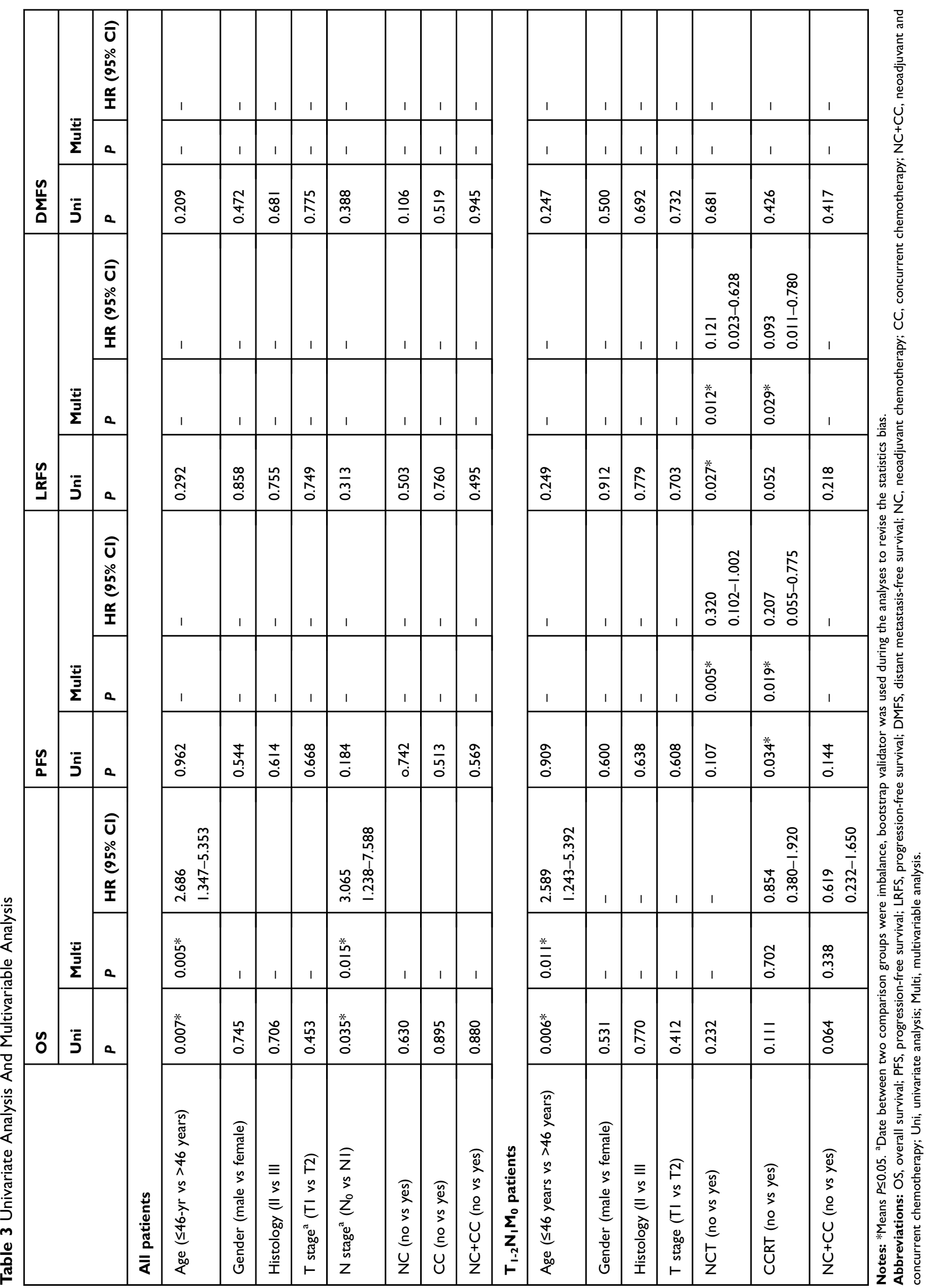


A

N stage

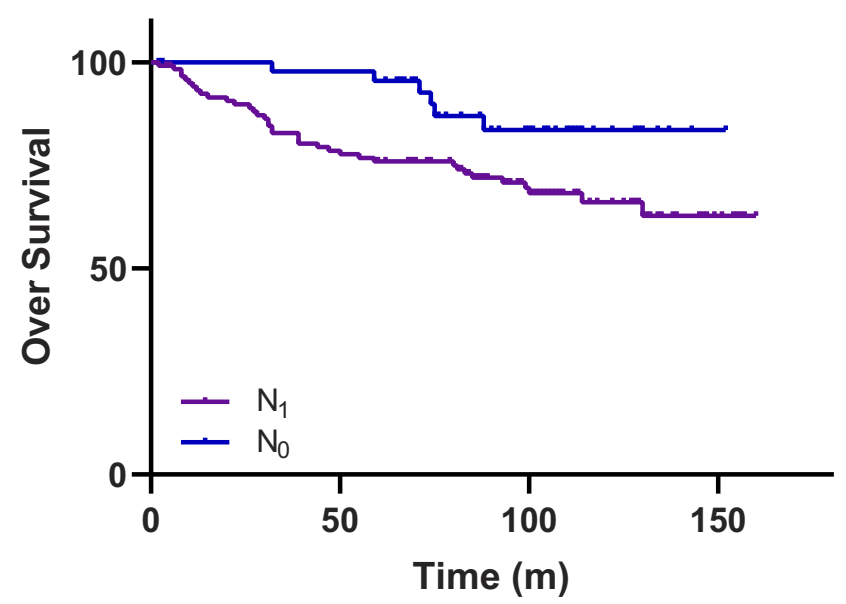

B

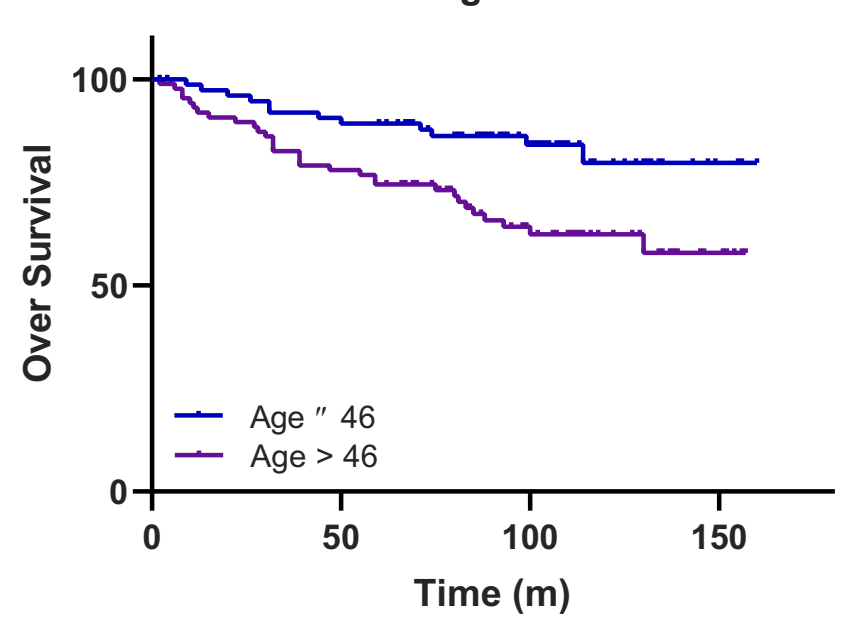

Figure $2 \mathrm{~N}$ stage and age were the independent prognostic factors for over survival (OS) of stage II NPC patients. (A) OS tended to decrease with $N_{\text {I }}$ stage $(P=0.005)$; $(B)$ OS tended to decrease with older age ( $>46$ years old) $(P=0.015)$.

protection. In other words, IMRT can improve tumor delineation and target coverage, resulting in excellent outcomes for stage II NPC. Meta-analyses of randomized studies have indicated that combining chemotherapy and RT increases 5year survival by $4 \%$ to $6 \%$ and reduces the risk of mortality by $18 \%{ }^{28}$ Nevertheless, the value of chemotherapy for stage II NPC is still unknown in the IMRT era. The main advantage of neoadjuvant chemotherapy is early eradication of micro-metastasis, which consequently enhances OS by reducing the distant metastasis rate. Due to both locoregional control and survival benefit, concurrent chemoradiotherapy is better than RT alone, which has been a standard treatment for locally advanced NPC. ${ }^{29,30}$ For earlystage treatment, Cheng et $\mathrm{al}^{31}$ reported that RT alone achieves a loco-regional control rate of $91.7 \%$ in patients (12) with stage I or II NPC and that concurrent
A PFS
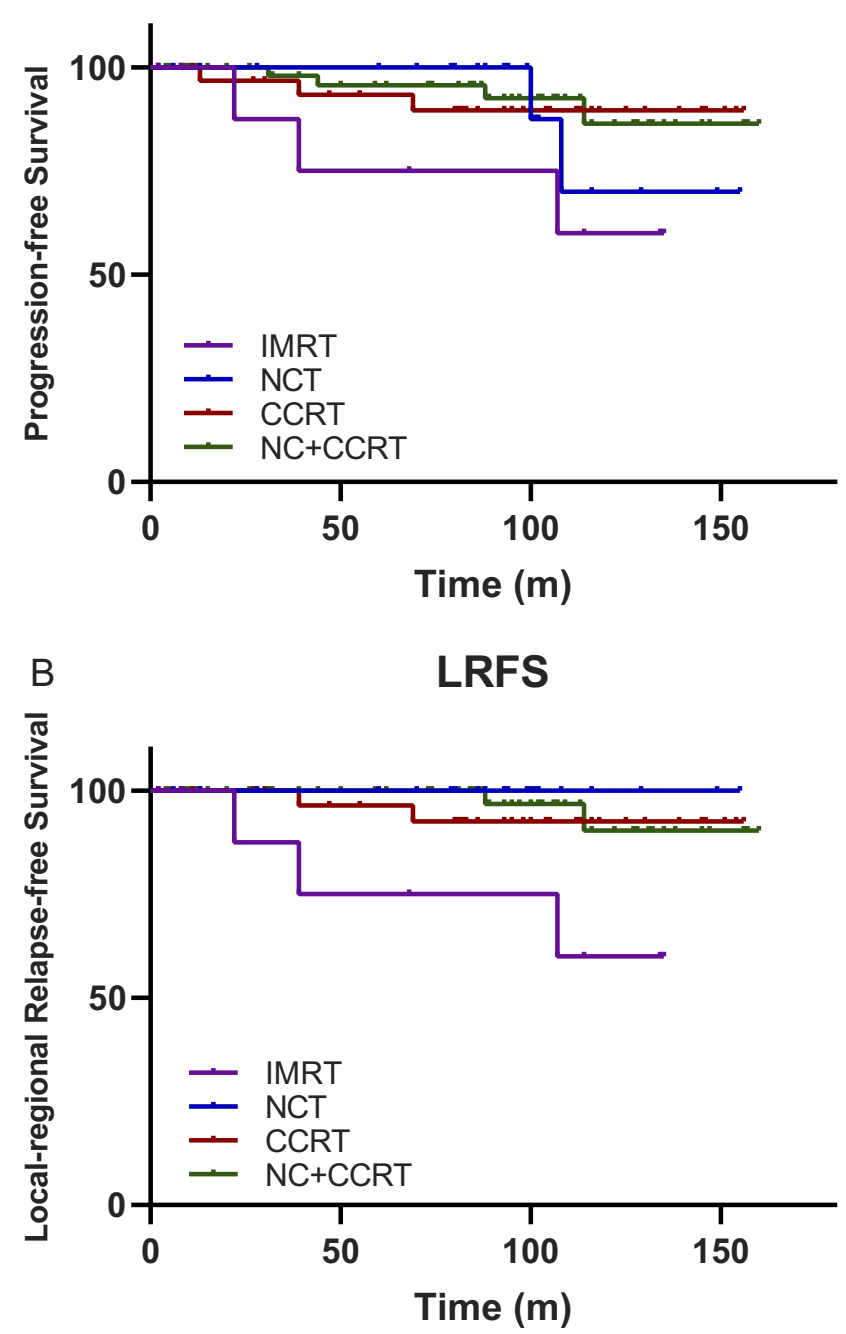

Figure 3 In $T_{1-2} N_{1} M_{0}$ group, intensity-modulated radiotherapy (IMRT) combined with chemotherapy had more loco-regional control effective. (A) Neoadjuvant chemotherapy followed by concurrent chemoradiotherapy (NC+CCRT) can improve progression-free survival significantly $(P=0.037)$; $(B)$ the addition of chemotherapy to IMRT can improve the local-regional relapse-free survival significantly (neoadjuvant chemotherapy with radiotherapy (NCT) vs IMRT, $P=0.02$, concurrent chemoradiotherapy (CCRT) vs IMRT, $P=0.034$; $N C+C C R T$ vs IMRT, $P=0.007$ ).

chemotherapy achieves $100 \%$ at 3 years in patients (32) with AJCC 1997 stage II NPC. Other clinical trials have produced similar results. ${ }^{9,25}$ However, the expected advantageous effect of neoadjuvant chemotherapy or concurrent chemotherapy was not found in the present study. The 3year OS, 5-year OS, 10-year OS, PFS, LRFS, and DMFS all had no significant differences between IMRT $+\mathrm{CT}$ groups and IMRT alone group, but they increased the acute toxicity reactions. Similar results were obtained in another recently reported retrospective study, ${ }^{32} 242$ stage II NPC patients in the study received IMRT combining with neoadjuvant chemotherapy and/or concurrent chemotherapy, the result 
Table 4 Acute Toxicity Reactions Between IMRT Alone, NCT, CCRT, NC+CCRT Groups

\begin{tabular}{|c|c|c|c|c|}
\hline & Number & $<$ Grade 3 & $\geq$ Grade 3 & $P$ \\
\hline & & $\begin{array}{l}\text { Number } \\
\text { (\%) }\end{array}$ & $\begin{array}{l}\text { Number } \\
\text { (\%) }\end{array}$ & \\
\hline All patients & 169 & & & \\
\hline $\begin{array}{l}\text { Hematotoxicity } \\
\text { IMRT alone } \\
\text { NCT } \\
\text { CCRT } \\
\text { NCT+CCRT }\end{array}$ & $\begin{array}{l}20 \\
25 \\
56 \\
68\end{array}$ & $\begin{array}{l}19(95) \\
18(72) \\
34(60.7) \\
39(57.4)\end{array}$ & $\begin{array}{l}\text { I (5) } \\
7(28) \\
22(39.3) \\
29(42.6)\end{array}$ & $0.007^{*}$ \\
\hline $\begin{array}{l}\text { Skin reaction } \\
\text { IMRT alone } \\
\text { NCT } \\
\text { CCRT } \\
\text { NCT+CCRT }\end{array}$ & $\begin{array}{l}20 \\
25 \\
56 \\
68\end{array}$ & $\begin{array}{l}19(95) \\
25(100) \\
50(89.3) \\
58(85.3)\end{array}$ & $\begin{array}{l}1(5) \\
0(0) \\
6(10.7) \\
10(14.7)\end{array}$ & 0.173 \\
\hline $\begin{array}{l}\text { Mucous reaction } \\
\text { IMRT alone } \\
\text { NCT } \\
\text { CCRT } \\
\text { NCT+CCRT }\end{array}$ & $\begin{array}{l}20 \\
25 \\
56 \\
68\end{array}$ & $\begin{array}{l}15(75) \\
21(84) \\
35(62.5) \\
38(55.9)\end{array}$ & $\begin{array}{l}5(25) \\
4(16) \\
21(37.5) \\
30(44.1)\end{array}$ & $0.049 *$ \\
\hline$T_{1-2} N_{1} M_{0}$ patients & 122 & & & \\
\hline $\begin{array}{l}\text { Hematotoxicity } \\
\text { IMRT alone } \\
\text { NCT } \\
\text { CCRT } \\
\text { NCT+CCRT }\end{array}$ & $\begin{array}{l}9 \\
21 \\
35 \\
57\end{array}$ & $\begin{array}{l}8(88.9) \\
18(85.7) \\
21(60) \\
34(59.6)\end{array}$ & $\begin{array}{l}\text { I (II.I) } \\
3(14.3) \\
14(40) \\
23(40.4)\end{array}$ & $0.033^{*}$ \\
\hline $\begin{array}{l}\text { Skin reaction } \\
\text { IMRT alone } \\
\text { NCT } \\
\text { CCRT } \\
\text { NCT+CCRT }\end{array}$ & $\begin{array}{l}9 \\
21 \\
35 \\
57\end{array}$ & $\begin{array}{l}8(88.9) \\
21(100) \\
31(88.6) \\
48(84.2)\end{array}$ & $\begin{array}{l}1 \text { (II.I) } \\
0(0) \\
4(11.4) \\
9(15.8)\end{array}$ & 0.435 \\
\hline $\begin{array}{l}\text { Mucous reaction } \\
\text { IMRT alone } \\
\text { NCT } \\
\text { CCRT } \\
\text { NCT+CCRT }\end{array}$ & $\begin{array}{l}9 \\
21 \\
35 \\
57\end{array}$ & $\begin{array}{l}7(77.8) \\
17(81) \\
21(60) \\
25(43.9)\end{array}$ & $\begin{array}{l}2(22.2) \\
4(19) \\
14(40) \\
32(56.1)\end{array}$ & $0.022 *$ \\
\hline
\end{tabular}

Note: *Means $P \leq 0.05$.

Abbreviations: IMRT, intensity-modulated radiotherapy; NCT, neoadjuvant chemotherapy with intensity-modulated radiotherapy; CCRT, concurrent chemotherapy with intensity-modulated radiotherapy; $\mathrm{NC}+\mathrm{CCRT}$, neoadjuvant and concurrent chemotherapy with intensity-modulated radiotherapy.

showed that combined regimens did not improve 5-year OS, PFS, LRFS, and DMFS, but were associated with higher incidences of acute toxicity reactions than IMRT alone.

The disappointing results of chemotherapy for stage II NPC patients may have several explanations. First, distant metastasis was the most common pattern of treatment failure. Significantly more patients with $\mathrm{N}_{1}$ stage and
$\mathrm{T}_{2} \mathrm{~N}_{1} \mathrm{M}_{0}$ stage were in the IMRT $+\mathrm{CT}$ groups compared to the IMRT alone group (both $P=0.001$ ), which may explain the inferior prognosis in the IMRT $+\mathrm{CT}$ groups. For patients with stage II, several studies have demonstrated that stage $\mathrm{N}_{1}$ is thegreatestisk factor in predicting DMFS and OS. Luo et $\mathrm{al}^{23}$ reported that patients with stage $\mathrm{T}_{1-2} \mathrm{~N}_{1} \mathrm{M}_{0}$ have greater 5-year accumulated distant metastasis rates than those with stage $\mathrm{T}_{1-2} \mathrm{~N}_{0} \mathrm{M}_{0}$, and they also showed that OS is significantly higher in the $\mathrm{T}_{2} \mathrm{~N}_{0} \mathrm{M}_{0}$ group than the $\mathrm{T}_{1-2} \mathrm{~N}_{1} \mathrm{M}_{0}$ group $(P=0.028)$. Similarly, all $5(6.5 \%)$ patients who developed distant metastasis belonged to stage $\mathrm{T}_{1-2} \mathrm{~N}_{1} \mathrm{M}_{0}$ in the present study. Additionally, $\mathrm{N}$ stage was an independent prognosis of increasing risk of OS $(P=0.015)$. Together, these conclusions suggest that $\mathrm{T}_{1-2} \mathrm{~N}_{1} \mathrm{M}_{0}$ is a unique subgroup with treatment outcomes far from satisfactory. Therefore, it is reasonable to presume that stage $T_{1-2} \mathrm{~N}_{1} \mathrm{M}_{0}$ NPC may need a more intensive treatment modality than stage $T_{2} \mathrm{~N}_{0} \mathrm{M}_{0}$ NPC. For $\mathrm{T}_{1-2} \mathrm{~N}_{1} \mathrm{M}_{0}$ NPC patients in the subgroup analysis, adding chemotherapy to IMRT favored loco-regional control, but it did not improve OS or prevent distant failure. Based on these findings, neoadjuvant chemotherapy and concurrent chemotherapy can both be expected to improve local-regional control in $\mathrm{T}_{1-2} \mathrm{~N}_{1} \mathrm{M}_{0}$ NPC patients, but this expectation was not reflected by an OS improvement.

Second, the potential effect of chemotherapy on eliminating distant metastasis could be diluted by an uncontrolled loco-regional tumor. In the present study, four patients (4/5) developed metastasis synchronously or after loco-regional recurrence. DMFS was still stable in the third year $(98.7 \%)$, fifth year $(97.9 \%)$, and tenth year $(94.8 \%)$, indicating that distant metastasis mostly occurred in the first 3 years. The important finding for stage II NPC was different from advanced NPC, in which distant metastasis mainly occurred after 3 years. ${ }^{3}$ Because IMRT provides a sufficient dose to the tumor target and kills micro-foci in early-stage NPC, chemotherapy probably reaches the highest efficacy with the combined modality of IMRT in stage II NPC. However, there was no room for improving local tumor control or to decrease distant metastases. With gradually decreasing PFS, LRFS and DMFS rates, the OS rate greatly decreased (14.3\%) from the third year to tenth year in the study. These results also suggested that death was mainly caused by cancer indirectly early on, while the reasons of death later on were mainly related to noncancer effects, including treatment-related toxicities, second primary tumor, natural deaths or other diseases, which agreed with 
the opinions of others. ${ }^{3,33}$ Therefore, longer follow-up periods of early-stage NPC patients resulted in lower potential effects of chemotherapy.

Finally, using neoadjuvant or concurrent chemotherapy can enhance radio-sensitivity in the treatment of NPC. However, delayed administration of RT may cause accelerated repopulation of tumor clones, which may contribute to lower LRFS in the neoadjuvant chemotherapy group. Acute toxicities associated with chemotherapy may decrease the survival benefit, which agreed with the conclusions of Guo et $\mathrm{al}^{24}$ and $\mathrm{Xu}$ et $\mathrm{al}^{34}$ Similar to previous studies, leucopenia, neutropenia, thrombocytopenia, skin reaction, and mucous reaction were mainly observed in patients. Compared to the IMRT alone group, the degrees of acute treatment-associated toxicities were notably higher in the IMRT+CT groups in the present study. There were more patients who suffered $\geq$ grade 3 acute hematologic toxicities and mucous reaction in the chemotherapy (including NCT, CCRT, NT+CCRT) groups, which was consistent with previous studies. ${ }^{23,35}$ Because the chemotherapy was untargeted, the combination of chemotherapy and IMRT not only attacked tumor cells but also normal cells, indicating that the balance between disease and human body was disturbed. This disadvantage may compromise the advantage of killing micrometastasis in the early phase. Therefore, further studies on combining high efficiency and low toxicity medicines, target therapy or immunotherapy with IMRT should be conducted to improve the OS of patients with stage II NPC.

The present study has several limitations. First, the number of IMRT alone patients was limited. Second, late toxicity reactions could not be completely collected for analysis. Third, during the long follow-up period, the chemotherapy regimens were not unified, which may influence the results. Therefore, well-designed randomized trials with long-term follow-up are important in the future.

\section{Conclusion}

In conclusion, patients with stage II NPC treated with IMRT obtained satisfactory survival outcomes. Moreover, the addition of chemotherapy did not further improve the survival of stage II NPC patients treated with IMRT. Almost all disease progress developed within 3 years after treatment, which differs from advanced NPC. Chemotherapy can improve local-regional control in $\mathrm{T}_{1-2} \mathrm{~N}_{1} \mathrm{M}_{0}$ NPC patients, but it does not improve OS. Furthermore, the addition of chemotherapy increased the acute toxicity reactions, especially hematotoxicity and mucous reaction. In the future, additional data from randomized trials are still urgently needed to guide the management of stage II NPC patients.

\section{Acknowledgments}

This work was supported by the Key Research and Development Plan of Shandong, China (grant no. 2019GGX101057), National key research and development program of China (grant no. 2018YFC1313200), and Science Technology Program of Jinan (grant no. 201821011).

\section{Disclosure}

The authors report no conflicts of interest in this work.

\section{References}

1. Cao SM, Simons MJ, Qian CN. The prevalence and prevention of nasopharyngeal carcinoma in China. Chin J Cancer. 2011;30(2):114119.

2. Chua DT, Ma J, Sham JS, et al. Improvement of survival after addition of induction chemotherapy to radiotherapy in patients with early-stage nasopharyngeal carcinoma: subgroup analysis of two Phase III trials. Int J Radiat Oncol Biol Phys. 2006;65(5):13001306. doi:10.1016/j.ijrobp.2006.02.016

3. Wu LR, Liu YT, Jiang N. et al. Ten-year survival outcomes for patients with nasopharyngeal carcinoma receiving intensity-modulated radiotherapy: an analysis of 614 patients from a single center. Oral Oncol;2017. 26-32. doi:10.1016/j.oraloncology.2017.03.015

4. Chen Q, Han L, Lu J, et al. Sequential chemotherapy and intensitymodulated radiation therapy in the management of locoregionally advanced nasopharyngeal carcinoma: experience of 370 consecutive cases. BMC Cancer. 2010;10(1):39. doi:10.1186/1471-2407-10-663

5. Al-Sarraf M, LeBlanc M, Giri PG, et al. Chemoradiotherapy versus radiotherapy in patients with advanced nasopharyngeal cancer: phase III randomized Intergroup study 0099. J Clin Oncol. 1998;16 (4):1310-1317. doi:10.1200/JCO.1998.16.4.1310

6. Wang M, Tian H, Li G, et al. Significant benefits of adding neoadjuvant chemotherapy before concurrent chemoradiotherapy for locoregionally advanced nasopharyngeal carcinoma: a meta-analysis of randomized controlled trials. Oncotarget. 2016;7(30):48375-48390. doi:10.18632/oncotarget.10237

7. Cheng SH, Tsai SY, Yen KL, et al. Concomitant radiotherapy and chemotherapy for early-stage nasopharyngeal carcinoma. J Clin Oncol Of J Am Soci Clin Oncol. 2000;18(10):2040. doi:10.1200/ JCO.2000.18.10.2059

8. Xiao WW, Han F, Lu TX, et al. Treatment outcomes after radiotherapy alone for patients with early-stage nasopharyngeal carcinoma. Int $J$ Radiat Oncol Biol Phys. 2009;74(4):1070-1076. doi:10.1016/j.ijrobp.2008.09.008

9. Xu T, Hu C, Wang X, et al. Role of chemoradiotherapy in intermediate prognosis nasopharyngeal carcinoma. Oral Oncol. 2011;47 (5):408-413. doi:10.1016/j.oraloncology.2011.03.008

10. Lee N, Xia P, Quivey JM, et al. Intensity-modulated radiotherapy in the treatment of nasopharyngeal carcinoma: an update of the UCSF experience. Int $J$ Radiat Oncol Biol Phys. 2002;53(1):12-22. doi:10.1016/s0360-3016(02)02724-4

11. Kam MK, Teo PM, Chau RM, et al. Treatment of nasopharyngeal carcinoma with intensity-modulated radiotherapy: the Hong Kong experience. Int J Radiat Oncol Biol Phys. 2004;60(5):1440-1450. doi:10.1016/j.ijrobp.2004.05.022 
12. Tham IW, Lin S, Pan J, et al. Intensity-modulated radiation therapy without concurrent chemotherapy for stage IIb nasopharyngeal cancer. Am J Clin Oncol. 2010;33(3):294-299. doi:10.1097/COC.0b0 $13 \mathrm{e} 3181 \mathrm{~d} 2 \mathrm{edab}$

13. Su SF, Han F, Zhao C, et al. Long-term outcomes of early-stage nasopharyngeal carcinoma patients treated with intensity-modulated radiotherapy alone. Int J Radiat Oncol Biol Phys. 2012;82(1):327333. doi:10.1016/j.ijrobp.2010.09.011

14. Chen K, Zhu X, Li L, et al. Comparison of the efficacy between concurrent chemoradiotherapy with or without adjuvant chemotherapy and intensitymodulated radiotherapy alone for stage II nasopharyngeal carcinoma. Oncotarget. 2016;7(42):69041-69050. doi:10.18632/oncotarget.11978

15. Su Z, Mao YP, Tang J, et al. Long-term outcomes of concurrent chemoradiotherapy versus radiotherapy alone in stage II nasopharyngeal carcinoma treated with IMRT: a retrospective study. Tumor Biol. 2016;37(4):4429-4438. doi:10.1007/s13277-015-4266-5

16. Zhang LN, Gao YH, Lan XW, et al. Propensity score matching analysis of cisplatin-based concurrent chemotherapy in low risk nasopharyngeal carcinoma in the intensity-modulated radiotherapy era. Oncotarget. 2015;6(41):44019-44029. doi:10.18632/oncotarget.5806

17. Joseph W, Eng Huat T, Bee Choo T, et al. Randomized trial of radiotherapy versus concurrent chemoradiotherapy followed by adjuvant chemotherapy in patients with American Joint Committee on Cancer/International Union against cancer stage III and IV nasopharyngeal cancer of the endemic variety. J Clin Oncol Of J Am Soci Clin Oncol. 2005;23(27):6730-6738. doi:10.1200/JCO.2005.16.790

18. Al-Sarraf M, Leblanc M, Giri PG, et al. Chemoradiotherapy versus radiotherapy in patients with advanced nasopharyngeal cancer: phase III randomized Intergroup study 0099. J Clin Oncol. 1998;16 (4):1310-1317. doi:10.1200/JCO.1998.16.4.1310

19. Chen QY, Wen YF, Guo L, et al. Concurrent chemoradiotherapy vs radiotherapy alone in stage II nasopharyngeal carcinoma: phase III randomized trial. J Natl Cancer Inst. 2011;103(23):1761-1770. doi:10.1093/jnci/djr432

20. Adelstein D, Gillison ML, Pfister DG, et al. NCCN guidelines insights: head and neck cancers, version 2.2017. J Natl Compr Canc Netw. 2017;15(6):761-770. doi:10.6004/jnccn.2017.0101

21. Chua DTT, Ma J, Sham JST, et al. Long-term survival after cisplatinbased induction chemotherapy and radiotherapy for nasopharyngeal carcinoma: a pooled data analysis of two phase III trials. J Clin Oncol Of J Am Soci Clin Oncol. 2005;23(6):1118-1124. doi:10.1200/ JCO.2005.12.081

22. Lin S, Lu JJ, Han L, et al. Sequential chemotherapy and intensitymodulated radiation therapy in the management of locoregionally advanced nasopharyngeal carcinoma: experience of 370 consecutive cases. BMC Cancer. 2010;10:39. doi:10.1186/1471-2407-10-663
23. Luo S, Zhao L, Wang J, et al. Clinical outcomes for early-stage nasopharyngeal carcinoma with predominantly WHO II histology treated by intensity-modulated radiation therapy with or without chemotherapy in nonendemic region of China. Head Neck. 2014;36 (6):841-847. doi:10.1002/hed.23386

24. Guo Q, Lu T, Lin S, et al. Long-term survival of nasopharyngeal carcinoma patients with Stage II in intensity-modulated radiation therapy era. Jpn J Clin Oncol. 2016;46(3):241-247. doi:10.1093/jjco/hyv192

25. Kang MK, Oh D, Cho KH, et al. ERRATUM: role of chemotherapy in stage II nasopharyngeal carcinoma treated with curative radiotherapy. Cancer Res Treat Off J Korean Cancer Assoc. 2016;48 (1):425. doi: $10.4143 / \mathrm{crt} .2014 .141 .2$

26. Efron B. The Jackknife, the Bootstrap, and Other Resampling Plans [M]. Philadelphia: SIAM; 1982.

27. Lin JC, Jan JS, Hsu CY, et al. Phase III study of concurrent chemoradiotherapy versus radiotherapy alone for advanced nasopharyngeal carcinoma: positive effect on overall and progression-free survival. $J$ Clin Oncol. 2003;21:631-637. doi:10.1200/JCO.2003.06.158

28. Chua DT, Sham JS, Kwong DL, Au GK. Treatment outcome after radiotherapy alone for patients with Stage I-II nasopharyngeal carcinoma. Cancer. 2010;98(1):74-80. doi:10.1002/cncr.11485

29. Andersson-Anvret M, Forsby N, Klein G. Nasopharyngeal carcinoma. Prog Exp Tumor Res. 1978;21(1):100.

30. Katano A, Takahashi W, Yamashita H, et al. Radiotherapy alone and with concurrent chemotherapy for nasopharyngeal carcinoma: a retrospective study. Medicine (Baltimore). 2018;97(18):e0502. doi:10.1097/MD.0000000000010502

31. Cheng SH, Tsai SY, Yen KL, et al. Concomitant radiotherapy and chemotherapy for early-stage nasopharyngeal carcinoma. J Clin Oncol Of J Am Soci Clin Oncol. 2000;18(10):2040. doi:10.1200/ JCO.2000.18.10.2059

32. Fangzheng W, Chuner J, Quanquan S, et al. Addition of chemotherapy to intensity-modulated radiotherapy does not improve survival in stage II nasopharyngeal carcinoma patients. J Cancer. 2018;9 (11):2030-2037. doi:10.7150/jca.25042

33. Lee AWM, Poon YF, Foo W, et al. Retrospective analysis of 5037 patients with nasopharyngeal carcinoma treated during 1976-1985: overall survival and patterns of failure. Int J Radiat Oncol Biol Phys. 1992;23(2):261-270. doi:10.1016/0360-3016(92)90740-9

34. Xu T, Shen C, Zhu G, Hu C. Omission of chemotherapy in early stage nasopharyngeal carcinoma treated with IMRT: a paired cohort study. Medicine. 2015;94(39):e1457. doi:10.1097/MD.0000000000000874

35. Chen KH, Zhu XD, Li L, et al. Comparison of the efficacy between concurrent chemoradiotherapy with or without adjuvant chemotherapy and intensity-modulated radiotherapy alone for stage II nasopharyngeal carcinoma. Oncotarget. 2016;7(42):69041-69050. doi:10.18 632/oncotarget. 11978

\section{Publish your work in this journal}

Cancer Management and Research is an international, peer-reviewed open access journal focusing on cancer research and the optimal use of preventative and integrated treatment interventions to achieve improved outcomes, enhanced survival and quality of life for the cancer patient.
The manuscript management system is completely online and includes a very quick and fair peer-review system, which is all easy to use. Visit http://www.dovepress.com/testimonials.php to read real quotes from published authors. 